\title{
Josephson charge qubits: a brief review
}

\author{
Yu. A. Pashkin - O. Astafiev - T. Yamamoto • \\ Y. Nakamura · J. S. Tsai
}

Published online: 13 February 2009

(C) The Author(s) 2009. This article is published with open access at Springerlink.com

\begin{abstract}
The field of solid-state quantum computation is expanding rapidly initiated by our original charge qubit demonstrations. Various types of solid-state qubits are being studied, and their coherent properties are improving. The goal of this review is to summarize achievements on Josephson charge qubits. We cover the results obtained in our joint group of NEC Nano Electronics Research Laboratories and RIKEN Advanced Science Institute, also referring to the works done by other groups. Starting from a short introduction, we describe the principle of the Josephson charge qubit, its manipulation and readout. We proceed with coupling of two charge qubits and implementation of a logic gate. We also discuss decoherence issues. Finally, we show how a charge qubit can be used as an artificial atom coupled to a resonator to demonstrate lasing action.
\end{abstract}

Keywords Cooper pair box · Josephson charge qubit - Quantum state manipulation · Single-shot readout · Quantum coherence · Decoherence · Dephasing · Energy relaxation · Quantum logic gate · Artificial atom · Lasing

PACS 03.65.Yz $\cdot 03.67 . \mathrm{Lx} \cdot 42.50 . \mathrm{Pq} \cdot 73.23 . \mathrm{Hk} \cdot 85.25 . \mathrm{Cp} \cdot 85.35 . \mathrm{Gv}$

\section{Introduction}

By holding a promise of solving certain computational problems exponentially faster in comparison to the classical computers, the idea of quantum information processing [1] is pushing research on various physical quantum objects that can be used as

Yu. A. Pashkin—On leave from Lebedev Physical Institute, Leninskii Prospekt 53, Moscow 119991, Russia.

Yu. A. Pashkin $(\varangle) \cdot$ O. Astafiev · T. Yamamoto · Y. Nakamura · J. S. Tsai

NEC Nano Electronics Research Laboratories and RIKEN Advanced Science Institute,

34 Miyukigaoka, Tsukuba, Ibaraki 305-8501, Japan

e-mail: pashkin@frl.cl.nec.co.jp 
quantum bits, or qubits. From a purely mathematical discipline a few years ago, quantum computation has turned into an active research field of modern physics attracting efforts of experimentalists from various areas [2]. A number of physical objects have been suggested as potential implementations of qubits. However, solid-state circuits, and superconducting circuits in particular [3,4], are of great interest as they offer scalability, i.e., a possibility of making circuits with a larger number of interacting qubits. Within the past few years, there has been substantial experimental progress with single solid-state qubits in which coherent manipulation of quantum states has been performed [5-14]. Superconducting qubits utilize charge [5-7] or flux [8] degrees of freedom, or energy levels quantization in a single current-biased Josephson junction $[9,10]$. Inter-qubit coupling [15-20] and also quantum logic gates [21-23] have been reported later. Further progress in the field slowed down due to obvious decoherence issues that are still to be overcome in order to implement a working quantum computer.

In this review, we focus on charge qubits. The logical qubit states correspond to different charge states that can be manipulated by means of external electrical signals and then measured by a properly coupled detector. Besides a superconducting version of the charge qubit, there is a semiconducting version [11], also based on the charge degree of freedom. However, it is not in the scope of this review and will not be discussed. In what follows, only Josephson, or superconducting, charge qubits will be described.

The Josephson charge qubit circuits satisfy basically all the criteria for the implementation of quantum computation put forward by DiVincenzo [24]. They offer single qubits, i.e., structures that can be approximated as quantum two-level systems. Such systems possess coherence, can be initialized and read out. Finally, logic gates can be constructed and gate operation can be implemented.

Fabrication of charge qubits is a rather matured technology. They are fabricated using the so-called angle deposition technique through a suspended mask [25], that was first introduced for photolithography and then applied to electron beam lithography to produce submicron-size tunnel junction with superb control of junction parameters and excellent reproducibility. The most common qubit material is aluminum, a superconductor with a bulk transition temperature of $1.2 \mathrm{~K}$. It oxidizes to form a tunnel barrier with a low leakage when exposed to oxygen.

\section{Josephson charge qubit—-the first solid-state qubit}

The Josephson charge qubit is based on a Cooper pair box [26,27]: a nanometer-scale superconducting island, which is connected via a Josephson junction to a large electrode, called a reservoir. With typical island dimensions $1000 \mathrm{~nm} \times 50 \mathrm{~nm} \times 20 \mathrm{~nm}$ (length $\times$ width $\times$ thickness), the number of conduction electrons is equal to $10^{7}-$ $10^{8}$. Because of superconductivity, they all form Cooper pairs and condense to a single macroscopic ground state, with a superconducting energy gap $\Delta$ opened in the density of states. The superconducting ground state is thus separated by $\Delta$ from the quasiparticle excitations, which is favorable for the coherence of the system. Besides Cooper pairs, the island can also contain quasiparticles. However, if the temperature is low, $k_{B} T \ll \Delta$, then the thermal excitation of quasiparticles is strongly suppressed. Here $k_{B}$ is the Boltzmann constant. The number of Cooper pairs in the box can be changed 
by the voltage $V_{g}$ applied to the gate coupled to the box through the gate capacitor $C_{g}$. Because of the Josephson coupling $E_{J}$ between the box and the reservoir, Cooper pairs can tunnel into or out of the box. These charges are transferred one by one due to Coulomb blockade as the charging energy $E_{c} \equiv e^{2} / 2 C$ exceeds $k_{B} T$. The Hamiltonian of the system can be written as

$$
H=\sum_{n} 4 E_{c}\left(n_{g}-n\right)^{2}|n\rangle\langle n|-\frac{E_{J}}{2} \sum_{n}(|n\rangle\langle n+1|+| n+1\rangle\langle n|)
$$

where $n_{g}=C_{g} V_{g} / 2 e$ is the normalized gate induced charge, $e$ is the electron charge, $n$ is an integer number of Cooper pairs. In the absence of the Josephson coupling between the Cooper pair box and the reservoir, Eq. 1 describes the electrostatic energy of the system represented by a set of parabolas each corresponding to a certain charge number state. Theoretically, there is an infinite number of charge states at a certain gate voltage corresponding to a different number of Cooper pairs in the box. The neighboring parabolas intersect at $n_{g}=1 / 2,3 / 2, \ldots$ where the electrostatic energy of two charge states differing by one Cooper pair is equal. When $E_{J}$ is switched on, Josephson tunneling couples charge states, the degeneracy is lifted and an energy gap opens in the energy spectrum. The energy bands are similar to those calculated for a current-biased small Josephson junction [28]. Because the whole energy spectrum is periodic in the gate induced charge, one can just work in the vicinity of one degeneracy point reducing the Hilbert space just to two states, $|0\rangle$ and $|1\rangle$. In this case the Hamiltonian of the system, after linearization, takes the form

$$
H=-\frac{1}{2} \delta E\left(n_{g}\right) \sigma_{z}-\frac{1}{2} E_{J} \sigma_{x}
$$

where $\delta E\left(n_{g}\right) \equiv 4 E_{c}\left(n_{g}-1 / 2\right)$ is the difference of the electrostatic energy between the states $|0\rangle$ and $|1\rangle$ and $\sigma_{z}$ and $\sigma_{x}$ are Pauli matrices. As was pointed out in Ref. [27], the Cooper pair box is completely analogous to a spin-1/2 particle in a fictitious magnetic field $\left[E_{J}, 0,-\delta E\left(n_{g}\right)\right]$. The two eigenstates of the Hamiltonian (2) are

$$
\begin{aligned}
& |g\rangle=\sin \theta / 2|0\rangle+\cos \theta / 2|1\rangle \\
& |e\rangle=\cos \theta / 2|0\rangle-\sin \theta / 2|1\rangle,
\end{aligned}
$$

where $|g\rangle$ and $|e\rangle$ denote the ground and excited states, respectively, and

$$
\theta=\arctan \left(E_{J} / \delta E\left(n_{g}\right)\right)
$$

One can see that at the degeneracy point $\left(\delta E\left(n_{g}\right)=0\right),|g\rangle=\frac{1}{\sqrt{2}}(|0\rangle+|1\rangle)$ and $|e\rangle=\frac{1}{\sqrt{2}}(|0\rangle-|1\rangle)$. If the system is shifted away from the degeneracy $\left(\left|\delta E\left(n_{g}\right)\right| \gg\right.$ $\left.E_{J}\right)$ then the eigenstates approach the charge states. The eigenstates get closer to the charge states for larger ratio $E_{c} / E_{J}$ and larger gate offset from the degeneracy point within the range $0<n_{g}<1$. The gap between the ground and excited states changes as $\Delta E=\sqrt{\delta E\left(n_{g}\right)^{2}+E_{J}^{2}}$ being minimal at the degeneracy. 
The ground-state properties of the Cooper pair box were studied in Ref. [27] using an electrometer as a readout device for charge state measurements, and a coherent superposition of charge states was observed.

The energy gap of the Cooper pair box was revealed in the spectroscopy measurements [29] by irradiating a device by microwaves and tracing the peak position of a photon-assisted Josephson-quasiparticle (JQP) current [30,31] as a function of the microwave frequency. In order to do transport measurement through the Cooper pair box, a high-resistance probe junction was attached to it and biased such that the JQP cycle becomes possible. The resistance of the probe junction is typically about $10 \mathrm{M} \Omega$ or higher so that the quasiparticle tunneling rate $\Gamma_{q p} \ll E_{J} / \hbar$, where $\hbar$ is the Planck's constant $h$ divided by $2 \pi$.

The device described in Refs. [27,29] was a prototype for solid-state qubits, and those experimental demonstrations laid the groundwork for the many subsequent experimental demonstrations of the coherent properties of the Cooper pair box. More details on coherent properties of the Cooper pair box can be found elsewhere [32].

The breakthrough in the solid-state quantum computation was the demonstration of coherent oscillations in a Cooper pair box [5]. For this, a circuit similar to the one described in Ref. [29] was utilized. Manipulation of charge states $|0\rangle$ and $|1\rangle$ was done by means of nonadiabatic pulses applied to the Cooper pair box. The qubit schematics are shown in Fig. 1. In addition to the dc-bias gate, a pulse gate with capacitance $C_{p}$ is added to the Cooper pair box. The normalized gate-induced charge is then $n_{g}=\left(C_{g} V_{g}+C_{p} V_{p}\right) / 2 e$. A high-frequency coaxial line and a sample package were designed [33] to deliver pulses with a length as short as 80 ps provided by the pulse-pattern generator.

The coherent quantum state manipulation is done in the following way. First, the system is prepared in state $|0\rangle$ by choosing a gate voltage to the left (negative detuning) of the degeneracy point. Then, a sharp pulse is applied that shifts the system nonadiabatically to the degeneracy point. Because $|0\rangle$ is no longer an eigenstate at degeneracy, the system starts to evolve during the pulse duration time $\Delta t$ according to

$$
|\psi(t)\rangle=\exp [-i H \Delta t / \hbar]|0\rangle
$$

where $H$ is defined by Eq. 2 with $\delta E\left(n_{g}\right)=0$. After the pulse is terminated, the system stays in the superposed state $\cos \left(\frac{E_{J} \Delta t}{2 \hbar}\right)|0\rangle+i \sin \left(\frac{E_{J} \Delta t}{2 \hbar}\right)|1\rangle$. The probability for

Fig. 1 Schematic diagram of a Josephson charge qubit. Black bar denotes a Cooper pair box

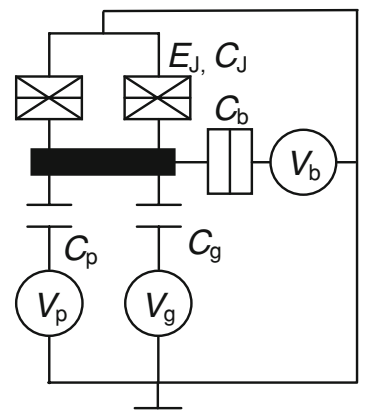


the system to be in state $|1\rangle$ after time $\Delta t$ is equal to $p(1)=\frac{1}{2}\left[1-\cos \left(\frac{E_{J} \Delta t}{\hbar}\right)\right]$ oscillating between 0 and 1 with a period $h / E_{J}$. Similarly, the probability $p(0)$ of being in state $|0\rangle$ oscillates with the same period but opposite phase. After the pulse, the state $|1\rangle$ is an excited state and should relax to the ground state. The readout process must be done before the relaxation takes place. Alternatively, this relaxation process itself can be used for the final state readout as this relaxation contains information about the final state. Our readout scheme is based on this relaxation process.

For the readout, we attach an additional electrode, called a probe, to the box through a tunnel junction. The probe is voltage-biased with an appropriate bias voltage $V_{b}$, so that two quasiparticles tunnel out sequentially only when there is one excess Cooper pair in the box. The bias voltage is chosen such that it supplies sufficient energy for the first and second quasiparticle to tunnel into the probe but not a third one [34]. Because of the charging effect, in the $|0\rangle$ state, the quasiparticle tunneling is Coulomb-blockaded. Therefore, by using this quasiparticle tunneling process, we can distinguish the two charge states. Also, this process initializes the qubit state to $|0\rangle$ after the readout. The Cooper pair tunneling during the pulse duration time $\Delta t$ and the subsequent quasiparticle relaxation constitute the well known Josephson-quasiparticle cycle of the single-electron transistor, however, the pulse-induced JQP peak is shifted to the left from the dc JQP peak by $C_{p} V_{p} / 2 e$. Thus, the readout process is extremely simple. Alternative readout schemes include the switching of a large Josephson junction [6], a radio-frequency SET [7], dispersive measurements [35,36], as well as quantum capacitance readout $[37,38]$.

One JQP cycle produces two electrons in the probe and the resulting current must be measured by an appropriate detector. Detecting just two electrons seems a very challenging task. Instead, we use an ensemble-averaged measurement. An array of identical pulses is sent to the Cooper box with a repetition time $T_{r}>\left(\Gamma_{q p 1} \Gamma_{q p 2}\right)^{-1}$. The repeated manipulation and relaxation processes give rise to a pulse-induced JQP current ideally equal to $2 e / T_{r}$. For a typical repetition time of 16-64 ns the resulting current of 5-20 pA is relatively easy to measure. Thus, we map the oscillation of $p(1)$ onto the oscillation of the pulse induced current.

For the quantum-state control, one can also use an ac gate-voltage pulse producing Rabi oscillations, the technique commonly used in atomic physics and NMR physics. When the ac driving frequency matches the energy gap between the two eigenstates, coherent quantum-state evolution between the two states takes place involving the photon energy of the driving field. Due to technical reasons, we used in our experiments a pulse composed of continuous-wave microwaves and a dc pulse. The dc pulse shifts the bias point from off-resonance to on-resonance with the microwave frequency. The observed Rabi oscillation frequency depends, as expected, on the power of the ac driving field, and Rabi oscillations involving multiple photons also take place [39].

\section{Single-shot readout}

In the experiments described above, charge qubit states are measured by ensemble averaging over many events. Various alternative schemes allowing single-shot readout were tested by other groups $[6,36]$. We have developed and demonstrated experimentally a 
Fig. 2 a Scanning electron micrograph of the qubit and a single-shot readout circuit. Inset shows sequence of pulses. b Typical time trace of readout pulses (upper panel) and the SET current. "Ones" and "zeros" correspond to the charged and neutral trap, respectively
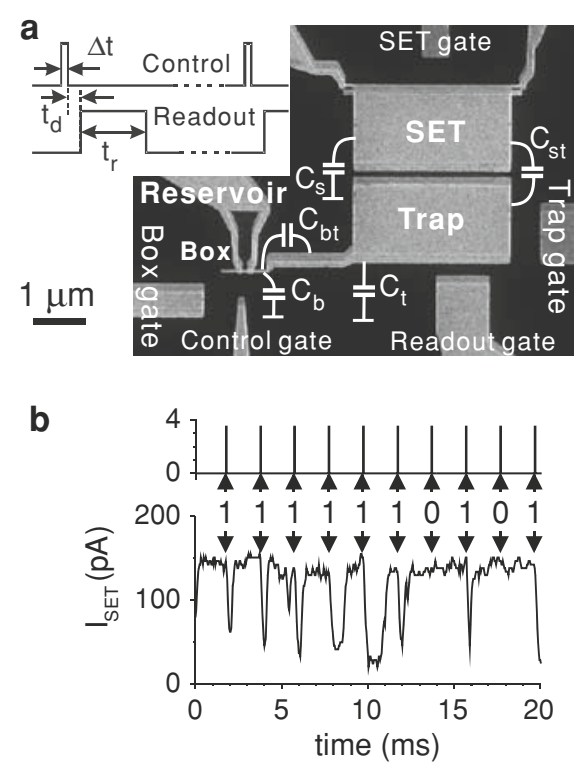

readout scheme that allows performing efficient single-shot measurement with a weak back-action on the qubit [40].

A scanning-electron micrograph of our circuit is shown in Fig. 2a. The device consists of a charge qubit and a readout circuit. The qubit is a Cooper-pair box described in Sect. 2 coupled to a reservoir through a Josephson junction. The reservoir is a big island with a large capacitance to the ground plane and galvanically isolated from the external environment. The readout part includes an electrometer, which is a conventional low-frequency single-electron transistor, and a charge trap placed in between the qubit and the SET. The trap is connected to the box through a high-resistance tunnel junction and capacitively coupled to the SET. The use of the trap enables us to separate in time coherent state manipulation and readout processes. Moreover, the qubit appears effectively decoupled from the SET.

The operation of the circuit can be described in the following way. During the qubit manipulation, the trap is kept unbiased prohibiting charge relaxation to the trap. Once the control pulse is terminated, the readout pulse (see the inset of Fig. 2a) is applied to the trap. The length and the amplitude of the readout pulse are adjusted (typically $300 \mathrm{~ns}, 3.5 e$ ) so that if there is an extra Cooper pair in the box after the control pulse termination, it escapes with a high probability to the trap through quasiparticle tunneling. After the charge is trapped, it remains in the trap for a long time (a reverse trap-to-box charge relaxation is suppressed due to the superconducting energy gap $2 \Delta$ ) and is measured by a low-frequency SET.

The qubit Hamiltonian is described by Eq. 2 and the manipulation is done in the way described in Sect. 2.

The upper panel of Fig. $2 \mathrm{~b}$ presents a sequence of readout pulses while the resulting time trace of the SET current is shown below. Negative switches of the SET current 
Fig. 3 Intensity plot showing coherent oscillations measured by averaging over many single-shot events

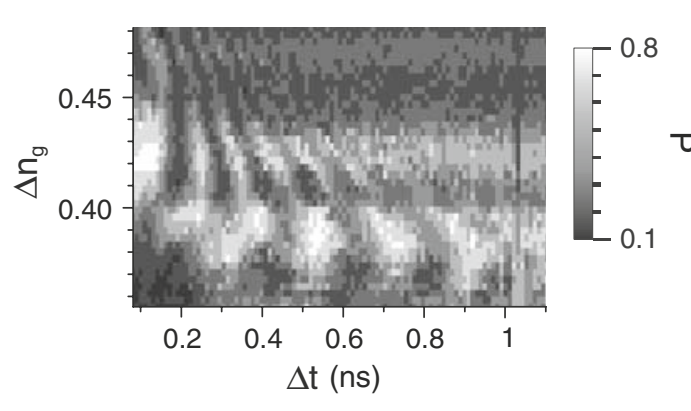

panel occurring simultaneously with the readout pulses are counted as charge detected events and marked as 1 . The missed events when no negative pulse is produced by the readout pulse are marked with 0 . The lifetime of the trapped charge is about $300 \mu \mathrm{s}$, and, therefore, the repetition time of $2 \mathrm{~ms}$ used in the experiment is sufficient for practically complete trap resetting. Then we count the number of detected switches $m$ (number of ones) and the total number of shots $n_{\text {tot }}$ (equal to the number of ones and zeros) and obtain the probability of charge detection as $P=m / n_{\text {tot }}$.

An experimentally obtained $P$ (normally, $n_{\text {tot }}=327$ is used per one experimentally measured $P$ ) as a function of the control pulse length $\Delta t$ and amplitude $\Delta n_{g}$ $\left(\equiv C_{g} V_{g} / 2 e\right)$ is shown as an intensity plot in Fig. 3. We define the pulse with $\Delta n_{g}=$ 0.42 and $\Delta t=120$ ps when $P$ reaches a maximum value, as a $\pi$-pulse.

Next, we measure the qubit characteristic relaxation times. We apply a control $\pi$-pulse to create state $|1\rangle$, but the readout pulse is applied with some delay, $t_{d}$. The probability $P$ is then measured as a function of $t_{d}$ and $t_{r} . P$ decays exponentially with $t_{d}$ presumably via energetically feasible Cooper pair tunneling $(1,0) \rightarrow(0,0)$, because alternative quasiparticle relaxation to the trap through the high resistive junction is blocked by $2 \Delta$ when the trap is not biased. The fit gives the relaxation rate to the reservoir $(220 \mathrm{~ns})^{-1}$. When no delay is introduced $\left(t_{d} \approx 0\right), P$ grows exponentially with $t_{r}$. This process is mainly determined by the quasiparticle decay to the trap with the rate $(37 \mathrm{~ns})^{-1} \gg(220 \mathrm{~ns})^{-1}$. Additionally, we obtain the rate of "dark" switches (false clicks) when the $|0\rangle$-state relaxes to the trap. These events can be presumably described by the process $(0,0) \rightarrow(-1,1)$, with a slow relaxation rate $(4100 \mathrm{~ns})^{-1}$ derived from the fit to the experimental data.

Our analysis shows that the efficiency of this readout scheme for states $|1\rangle$ and $|0\rangle$ is equal to $87 \%$ and $93 \%$, respectively.

\section{Decoherence in charge qubits}

Due to decoherence, the observed quantum coherent oscillations decay as a function of the pulse width. The oscillations last up to about $5 \mathrm{~ns}$ at the degeneracy point but are much shorter off-degeneracy indicating that the mechanisms of decoherence are different. Generally, two processes are relevant for the observed decoherence: energy relaxation and dephasing characterized by the corresponding time scales. We have tried to look closer into both mechanisms. 


\subsection{Free-induction decay and charge echo}

We have measured coherent oscillations away from the degeneracy point $\left(|\delta E| \gg E_{J}\right)$ [41]. For that purpose, we applied two short $(\Delta t=80 \mathrm{ps})$ pulses with a delay time $t_{d}$ in between. $\Delta t$ and $t_{d}$ were still much shorter than $1 / \Gamma_{q p 1}$. The height of the pulses was adjusted so that each pulse acts as a $(\pi / 2)_{x}$-pulse, which rotates the pseudo spin by 90 degrees around the $x$-axis. Starting from the ground state, the first $(\pi / 2)_{x}$-pulse creates an equal superposition of the two charge states. During the delay time, the qubit phase evolves because of the energy difference $\Delta E$ between the two eigenstates. Then the second $(\pi / 2)_{x}$-pulse projects the phase information on the $z$-axis, i.e., the readout axis. As a function of $t_{d}$, the $z$-component of the final state oscillates as $\cos \left(\Delta E t_{d} / \hbar\right)$. This is a direct analogy of the Ramsey interference experiments in atomic physics or free-induction-decay experiments in NMR [42]. In this experiment, the oscillating signal decays within a few-hundred picoseconds, much faster than that in the singlepulse experiment at the degeneracy point. This fact can be explained by dephasing due to charge fluctuations. Because of the energy-level anticrossing, $\Delta E$ is less sensitive to the charge fluctuations at the degeneracy point than at the off-degeneracy point. Thus, the dephasing due to the charge fluctuations is much weaker at the degeneracy point.

In the charge echo experiment [41], three pulses are applied to the qubit. A third pulse inserted between the two $(\pi / 2)_{x}$-pulses acts as a $(\pi)_{x}$-pulse, which flips the sign of the phase. As a result, the phase evolutions in the first $\left(t_{d 1}\right)$ and the second part $\left(t_{d 2}\right)$ of the delay time cancel each other and the effect of the fluctuations is eliminated as long as the fluctuation of the energy level is slow compared to the delay time. In fact, the echo signal is observed only when the two parts of the delay time are almost equal, and the echo signal survives up to about $5 \mathrm{~ns}$ when we change the delay time. The result suggests that the low-frequency part of the energy-level fluctuations is dominant in the qubit decoherence. This is not surprising because the qubit, like a single-electron transistor, is a charge sensitive device and hence affected by the background charge fluctuations.

As a source of the low-frequency energy-level fluctuations, the so-called background charge fluctuations are considered. $1 / f$ charge noise is very common in single-electron devices and is usually attributed to random motion of charges either in the substrate or the junction barrier, although the exact mechanism is still not known [43-45]. Indeed, the sample studied here also showed $1 / f$ noise when the dc current through the probe junction was measured. Because of the narrow bandwidth of the measurement setup, the $1 / f$ noise spectrum could be measured only below $100 \mathrm{~Hz}$. However, here we assume that the $1 / f$ noise spectrum extends to infinite frequency and estimate the dephasing of the qubit due to the noise. Under the assumption of gaussian fluctuations, the dephasing factor as a function of the delay time $\left(t_{d}=\tau\right)$ in the free-induction decay is given as

$$
\langle\exp i \varphi(\tau)\rangle=\exp \left[-\frac{1}{2 \hbar^{2}} \int_{\omega_{m}}^{\infty} d \omega S_{\Delta E(\omega)}\left(\frac{\sin (\omega \tau / 2)}{\omega / 2}\right)^{2}\right]
$$


Here $S_{\Delta E(\omega)}=\left(4 E_{c} / e\right)^{2} S_{Q}(\omega)$ is a power spectral density of the energy level fluctuations caused by the $1 / f$ charge fluctuations with a power spectral density $S_{Q}(\omega) \equiv$ $\alpha / \omega$ and $\omega_{m}$ is the low-frequency cutoff corresponding to the measurement time of $20 \mathrm{~ms}$ (which is actually the measurement time for one data point). Similarly, for the echo experiment with $t_{d 1}=t_{d 2}=\tau / 2$ we obtain

$$
\langle\exp i \varphi(\tau)\rangle=\exp \left[-\frac{1}{2 \hbar^{2}} \int_{\omega_{m}}^{\infty} d \omega S_{\Delta E(\omega)}\left(\frac{\sin ^{2}(\omega \tau / 4)}{\omega / 4}\right)^{2}\right]
$$

The charge echo technique partly eliminates the low-frequency contribution to the free-induction decay making coherence time longer.

The value of $\alpha$ derived from the charge echo experiment, $\left(0.64 \times 10^{-3} e\right)^{2}$, is close to $\alpha=\left(1.3 \times 10^{-3} e\right)^{2}$ obtained from transport measurement. This supports the idea that the background charge noise is the dominant source of dephasing at the qubit off-degeneracy point.

\subsection{Energy relaxation}

Energy relaxation in a charge qubit is measured with the single-shot read-out scheme described in Sect. 3.

Although decoherence of small Josephson circuits had been analyzed in a number of theoretical papers $[3,4]$ and relaxation of excited states of the charge qubits off the degeneracy point had been reported earlier [46], systematic studies of decoherence in charge qubits were missing. We should mention, however, that decoherence in some versions of charge qubits, namely quantronium and transmon, has been studied in greater detail [47-49]. We have measured the charge qubit energy relaxation in a wide range of the qubit parameters and found that it is caused by quantum noise with approximately linear frequency dependence. We propose a model in which the quantum noise is caused by charge fluctuators, which give both the classical $1 / f$ noise and the quantum $f$ noise.

The sample schematically shown in Fig. 4a consists of a qubit and a readout part described in Sect. 3 and the qubit manipulation is performed by nonadiabatic pulsing described in Sect. 2.

To measure energy relaxation dynamics of the excited state $|e\rangle$, we use a combination of the $\pi$-pulse and an additional adiabatic pulse (a pulse with slow rise and fall times satisfying the condition of $\hbar \mid d\left(\Delta E / d t \mid \ll E_{J}^{2}\right)$. The manipulation procedure, schematically shown in Fig. $4 b$, includes three sequential steps: first, a $\pi$-pulse is applied to the box to prepare excited state $|1\rangle$; second, an adiabatic pulse shifts the box along the excited energy band to a point $n_{g}=n_{g 0}+n_{g a}$ and keeps it there for a time interval $t_{a}$, where relaxation from the excited state $|e\rangle$ to the ground state $|g\rangle$ may occur; and third, the adiabatic pulse brings the box to the initial position $n_{g 0}$ converting the excited state $|e\rangle$ to the state $|1\rangle$ and the ground state $|g\rangle$ to state $|0\rangle$. After that the readout pulse is applied to the trap. One can study relaxation dynamics at a desired value of $n_{g}$ by measuring the probability of state $|1\rangle$ as a function of time $t_{a}$. 

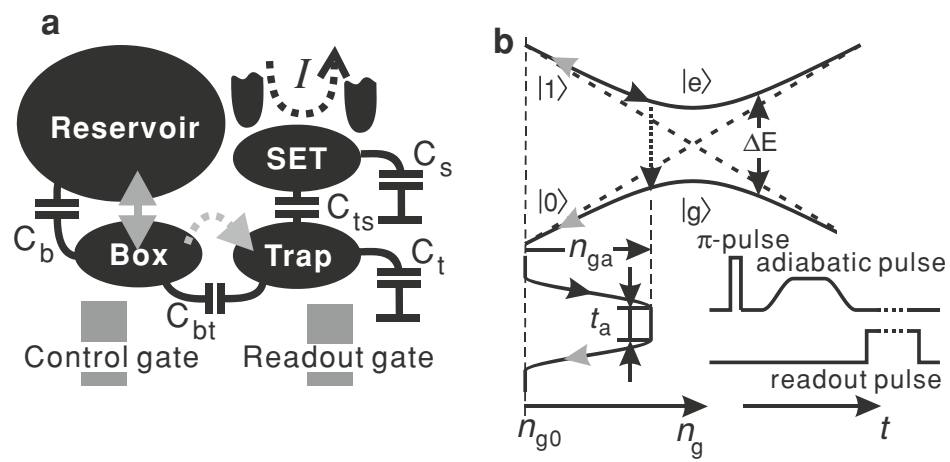

Fig. 4 a Schematics of the circuit to study energy relaxation. b Qubit energy bands and manipulation diagram

Fig. 5 Energy relaxation rate $\Gamma_{1}$ and dephasing rate $\Gamma_{2}$ as a function of the gate-induced charge $n_{g} . \Gamma_{2}$ was measured only at $n_{g}>0$ where coherent oscillations were observed

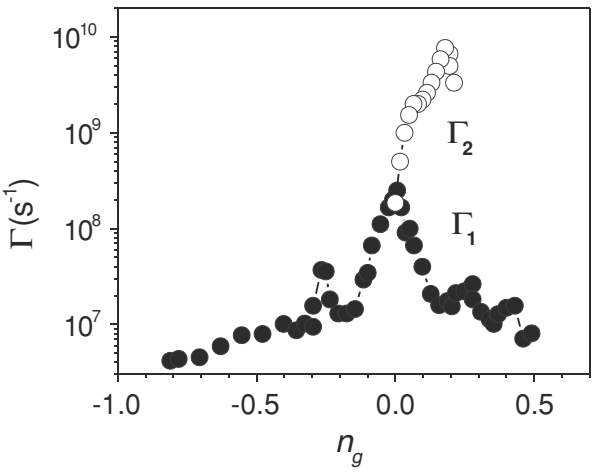

First, we measure the probability $P$ of the excited state $|1\rangle$ in the same way as described in Sect. 3. This is done for various initial gate charges $n_{g 0}$. From the fit, we obtain the energy relaxation rate $\Gamma_{1}$ as a function of $n_{g}$. Figure 5 shows $\Gamma_{1}$ (closed circles) as well as $\Gamma_{2}$ (open circles) derived from the decay of coherent oscillations.

Denoting the spectral density of energy fluctuation as $S_{\Delta E}(\omega)$, we express the relaxation rate as [50]

$$
\Gamma_{1}=\frac{\pi}{2 \hbar^{2}} S_{\Delta E}(\omega) \sin ^{2} \theta
$$

The overall behavior of $\Gamma_{1}$ in Fig. 5 ( $\Gamma_{1}$ decreases off the degeneracy point) is described by $\sin ^{2} \theta=E_{J}^{2} / E_{J}^{2}+\delta E^{2}$ ) characterizing the qubit coupling to the reservoir. The experimentally measured $\Gamma_{1}$ far from the degeneracy has a clear $E_{J}^{2}$ dependence as predicted by Eq. 8 when $\Delta E \gg E_{J}$. At the degeneracy point, $\Gamma_{1}=\pi S_{\Delta E}(\omega=$ $\left.E_{J} / \hbar\right) / 2 \hbar^{2}$ directly reproduces the frequency dependence of $S_{\Delta E}$. We argue that in our galvanically isolated device, the relaxation can not be explained by spontaneous emission to the remote environment coupled to the qubit through the electrical leads. Neither can it be explained by the effect of SET. 
Fig. 6 a Noise spectral density derived from $\Gamma_{1}$ for sample I (solid circles) and sample II (open circles and open triangles). Dashed-dotted line is $2 e^{2} R \hbar \omega$ ( $f$ noise) with $R=6 \Omega$. Dashed line is

$1 / f$ noise Eq. 9 with $\alpha=\left(1.3 \times 10^{-3} e\right)^{2}$ derived from $\Gamma_{2}$. Dotted line is a sum of $f$ and $1 / f$ noise

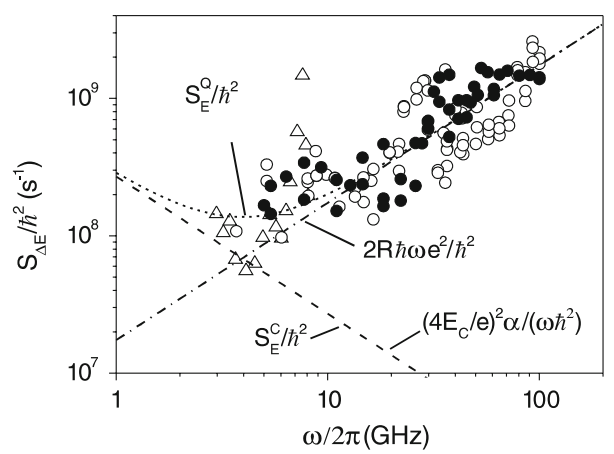

Let us now consider the effect of two-level fluctuators responsible for the low-frequency noise in nanoscale charge devices on the qubit relaxation. The charge noise produced by the fluctuators has a spectral density

$$
S_{q}(\omega)=\alpha /(2|\omega|)
$$

Note that the $1 / f$ noise is defined for both positive and negative frequencies. The parameter $\alpha$ has been obtained from the dc transport measurements for a device with essentially same geometry and fabrication process, and has been found to be $\alpha=\left(1.3 \times 10^{-3} e\right)^{2}$. This value is consistent with what was reported in other works [43-45], and also with the value derived from $T_{2}$.

Figure 6 summarizes the reduced noise spectrum $S_{\Delta E}$ derived from the measured $\Gamma_{1}$ according to Eq. 8 for two measured samples. Despite some scattering, $S_{\triangle E}$ exhibits a rise with $\omega$. The dashed-dotted line is a linear dependence (as in the case of some effective Ohmic environment), which we present in the form of $2 R \hbar \omega e^{2} / \hbar^{2}$ with $R=6 \Omega$. The actual rise of the experimental data is not monotonic but has some resonance-like peaks, for instance, at 7 and $30 \mathrm{GHz}$. This probably reflects coupling to some resonances that can be either two-level oscillators or just geometrical resonances in the sample package. $\Gamma_{1}$ approaches the $1 / f$ noise at low frequencies shown by the dashed line calculated with $\alpha=\left(1.3 \times 10^{-3} e\right)^{2}$ (see Eq. 9). Both $1 / f$ and $f$ dependencies cross at $\omega_{c}=2 \pi \times 2.6 \mathrm{GHz}$. This crossover frequency may formally relate to the effective temperature $T_{c}=\hbar \omega_{c} / k_{B}=120 \mathrm{mK}$, close to the electron temperature. This fact motivated us to propose the following model which may explain the measured noise spectra.

It is generally accepted that the low-frequency $1 / f$ noise is produced by a bath of two-level fluctuators at an effective temperature $T_{c}$ and weakly coupled to the qubit. We extend this concept to a higher frequency range also attributing the high-frequency $f$ noise to the bath of two-level fluctuators. In this model, pure dephasing is caused by the fluctuators in thermal equilibrium, switching between the two levels and producing the classical noise $S_{E}^{C}\left(S_{\Delta E}=\left(4 E_{c} / e\right)^{2} S_{q}\right)$. The energy relaxation is caused by the fluctuators, which have larger energy separation, staying in the ground state. Namely, the "hot" qubit $\left(\Delta E>k_{B} T_{c}\right)$ can only release energy $\Delta E$ to the "cold" fluctuator bath but cannot absorb energy from it. In other words, energy $\Delta E$ may activate 
fluctuators of the "cold" bath, that are "quiet" in equilibrium (do not contribute to $S_{\Delta E}^{C}$ but produce quantum noise $S_{\Delta E}^{Q}$. As we discussed above, $S_{\Delta E}^{C}$ is expected to have $1 / f$ dependence, while $S_{\triangle E}^{Q}$ is roughly proportional to $f$ according to our experimental data, and is consistent with the "Ohmic" picture. Such a simple model was recently supported by a more rigorous analysis [51].

Within this model one can predict the classical noise if the energy relaxation is known. For instance, if the high-frequency $\left(\omega \gg \omega_{c}\right) f$-part is $S_{q}^{Q}=\beta \hbar \omega$, the $1 / f$ classical noise can be found from the condition $\alpha / 2 \omega_{c}=\beta \hbar \omega_{c}$, where $\beta$ is a temperature independent parameter. The corresponding $1 / f$ noise is then

$$
S_{q}^{C}=\frac{\beta\left(k_{B} T_{c}\right)^{2}}{\hbar|\omega|} .
$$

As follows from Eq. 10, $\alpha=\beta\left(k T_{c}\right)^{2} / \hbar$ is expected to be proportional to $T_{c}^{2}$ because of the scale invariance. Indeed, $T^{2}$-dependence was observed in later experiments [52].

\section{Coupled charge qubits}

One of the key steps in constructing a quantum computer is to couple two qubits and then scale the circuit to many qubits. Qubit-qubit coupling that can be switched on and off on demand is required for making a quantum processor prototype. A few schemes for controllable coupling were proposed [53-55] and implemented [56].

We have performed experiments demonstrating for the first time quantum coherent dynamics of two solid-state qubits with constant coupling [15] as well as quantum logic gate operation [21].

\subsection{Coherent dynamics of two charge qubits}

The circuit we study [15] is shown schematically in Fig. 7. It consists of two charge qubits that are electrostatically coupled by an on-chip capacitor $C_{m}$. Both qubits have a common pulse gate but separate dc gates, probes and reservoirs. The pulse gate has nominally equal coupling to each box. In the experiment, both reservoirs are kept grounded. External controls that we have in the circuit are the dc probe voltages $V_{b 1}$ and $V_{b 2}$, dc gate voltages $V_{g 1}$ and $V_{g 2}$, and pulse gate voltage $V_{p}$. The information on the final states of the qubits after manipulation comes from the pulse-induced currents measured in the probes. From the transport measurements we obtain the characteristic energies: Cooper-pair charging energy of the first qubit $E_{c 1}=484 \mu \mathrm{eV}(117 \mathrm{GHz}$ in frequency units), Cooper-pair charging energy of the second qubit $E_{c 2}=628 \mu \mathrm{eV}$ $(152 \mathrm{GHz})$ and the coupling energy $E_{m}=65 \mu \mathrm{eV}(15.7 \mathrm{GHz})$. All these energies are higher than the energy of thermal fluctuations $k_{B} T \sim 3 \mu \mathrm{eV}(0.7 \mathrm{GHz})$. The charging and the coupling energies are defined as follows: $E_{c 1,2}=4 e^{2} C_{\Sigma 2,1} / 2\left(C_{\Sigma 1} C_{\Sigma 2}-C_{m}^{2}\right)$, where $C_{\Sigma 1,2}$ are the sum of all capacitances connected to the corresponding Cooper pair box including the coupling capacitance $C_{m}$, and $E_{m}=4 e^{2} C_{m} /\left(C_{\Sigma 1} C_{\Sigma 2}-C_{m}^{2}\right)$. 
Fig. 7 Schematic diagram of the two-coupled-qubit circuit. Black bars denote Cooper pair boxes

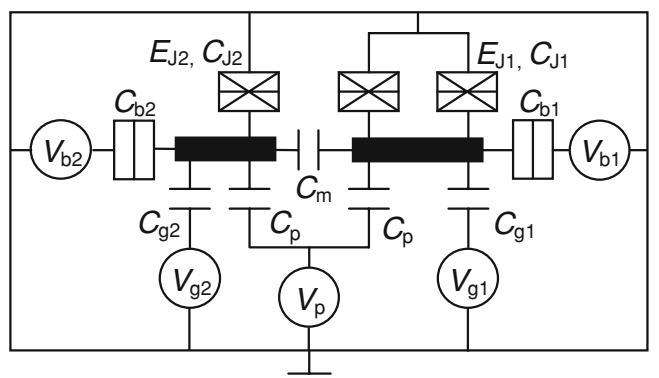

The qubits' Josephson energies $E_{J 1}$ and $E_{J 2}$ are determined from the period of coherent oscillations described later in the text.

The Hamiltonian of the system in the charge representation can be written as:

$$
\begin{aligned}
H= & \sum_{n_{1}, n_{2}}\left[E_{c 1}\left(n_{g 1}-n_{1}\right)^{2}+E_{c 2}\left(n_{g 2}-n_{2}\right)^{2}+E_{m}\left(n_{g 1}-n_{1}\right)\left(n_{g 2}-n_{2}\right)\right] \\
& \times\left|n_{1}, n_{2}\right\rangle\left\langle n_{1}, n_{2}\right|-\frac{E_{J 1}}{2}\left[\left|n_{1}, n_{2}\right\rangle\left\langle\left(n_{1}+1\right), n_{2}\right|\right. \\
& \left.+\left|n_{1},\left(n_{2}+1\right)\right\rangle\left\langle\left(n_{1}+1\right)\left(n_{2}+1\right)\right|\right]-\frac{E_{J 2}}{2}\left[\left|n_{1}, n_{2}\right\rangle\left\langle n_{1},\left(n_{2}+1\right)\right|\right. \\
& \left.+\left|\left(n_{1}+1\right), n_{2}\right\rangle\left\langle\left(n_{1}+1\right)\left(n_{2}+1\right)\right|\right] .
\end{aligned}
$$

Here $n_{1}$ and $n_{2}\left(n_{1}, n_{2}=0, \pm 1, \pm 2, \ldots\right)$ are the numbers of excess Cooper pairs in the first and the second Cooper pair boxes, and $n_{g 1,2}=\left(C_{g 1,2} V_{g 1,2}+C_{p} V_{p}\right) / 2 e$ are the normalized charges induced on the corresponding qubit by the dc and pulse gate electrodes. The eigenenergies, $E_{k}\left(n_{g 1}, n_{g 2}\right)(k=0,1,2, \ldots)$, of the Hamiltonian (1) form $2 e$-periodic energy bands corresponding to the ground $(k=0)$, first excited $(k=1)$, etc. states of the system. A contour plot of the ground-state energy band around zero gate-induced charge is shown in Fig. 8. It consists of hexagonal cells (shown partly) whose boundaries marked by the dashed lines delimit two neighboring charge states with equal electrostatic energies. For example, points R and L correspond to degeneracies between the states $|00\rangle$ and $|10\rangle$, and the states $|00\rangle$ and $|01\rangle$ that differ by one Cooper pair in the first (right) and the second (left) Cooper-pair box, respectively. The band has minima in the middle of each cell and also maxima in between (one of the maxima is marked by X). If we choose the dc gate charges $n_{g 1}$ and $n_{g 2}$ far from the boundaries but within the $(0,0)$ cell, then because of the large electrostatic energies we can assume that the system remains in the state $|00\rangle$. Strictly speaking, this charge stability diagram [57] is only valid in the absence of Josephson coupling; however, it also remains valid for small Josephson coupling, except at the boundaries where the charge states become superposed. Since the pulse gate has equal coupling to each qubit, the application of a pulse shifts the state of the system on this diagram along the line tilted at 45 degrees (indicated by arrows in Fig. 8). When the system is driven non-adiabatically to the point $\mathrm{R}$ or $\mathrm{L}$, it behaves like a single qubit oscillating between the degenerate states with a frequency $\omega_{1,2}=E_{J 1,2} / \hbar$. The final 
Fig. 8 Contour plot of the ground state energy band as a function of the normalized gate charges $n_{g 1}$ and $n_{g 2}$. Dashed lines are boundaries between charge states $\left(n_{1}, n_{2}\right)$ differing by one Cooper pair. The three thick arrows indicate non-adiabatic pulses used in the experiment

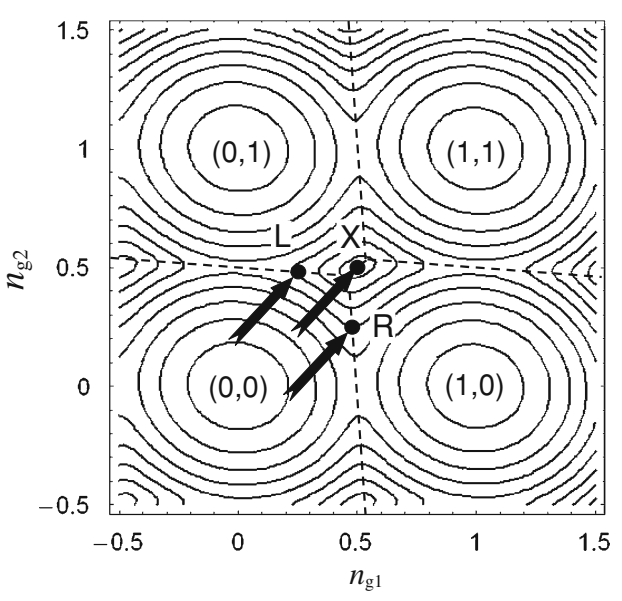

states are measured as average probe currents resulting from the decay of the excited states $(|10\rangle$ or $|01\rangle)$. From their oscillation period, we obtain $E_{J 1}$ and $E_{J 2}$.

Now let us consider point $\mathrm{X}$ in Fig. 8 which is a peak in the ground energy band. This point corresponds to two degeneracies: $E_{00}=E_{11}$ and $E_{10}=E_{01}$, and, therefore we call this a double degeneracy point. If the circuit is fabricated to have the following relation between the characteristic energies: $E_{J 1,2} \sim E_{m}<E_{c 1,2}$, then we can use a four-level approximation for the description of the system. The state of the system in the vicinity of $\mathrm{X}$ can be described by the coherent superposition of the four charge states $|00\rangle,|10\rangle,|10\rangle$ and $|11\rangle$ around $n_{g 1}=n_{g 2}=0.5$ while other charge states are separated by large energy gaps. These four charge states can be used as a new basis and the Hamiltonian (11) can be simplified to the following form:

$$
\begin{aligned}
H= & \sum_{n_{1}, n_{2}=0,1} E_{n 1 n 2}\left|n_{1}, n_{2}\right\rangle\left\langle n_{1}, n_{2}\left|-\frac{E_{J 1}}{2} \sum_{n_{2}=0,1}(|0\rangle\langle 1|+| 1\rangle\langle 0|)\right| n_{2}\right\rangle\left\langle n_{2}\right| \\
& -\frac{E_{J 2}}{2} \sum_{n_{1}=0,1}\left|n_{1}\right\rangle\left\langle n_{1}\right|(|0\rangle\langle 1|+| 1\rangle\langle 0|),
\end{aligned}
$$

where $E_{n 1 n 2}=E_{c 1}\left(n_{g 1}-n_{g 1}\right)^{2}+E_{c 2}\left(n_{g 2}-n_{2}\right)^{2}+E_{m}\left(n_{g 1}-n_{1}\right)\left(n_{g 2}-n_{2}\right)$ is the total electrostatic energy of the system $\left(n_{1}, n_{2}=0,1\right)$. The cross-section of the energy bands through the point $X$ is presented in Fig. 9. Here the dynamics of the quantum evolution becomes more complex (involving all four charge states) and reflects the coupling between the qubits. Exactly at the double degeneracy point, the time evolution of the system can be described analytically for any initial state. First, we write down the state of the system in general as:

$$
|\psi(t)\rangle=c_{1}|00\rangle+c_{2}|10\rangle+c_{3}|01\rangle+c_{4}|11\rangle
$$

where $c_{i}(i=1,2,3,4)$ are the time dependent probability amplitudes obeying the normalization condition $\sum_{i=1}^{4}\left|c_{i}\right|^{2}=1$ [58]. In the experiment, we measure probe 


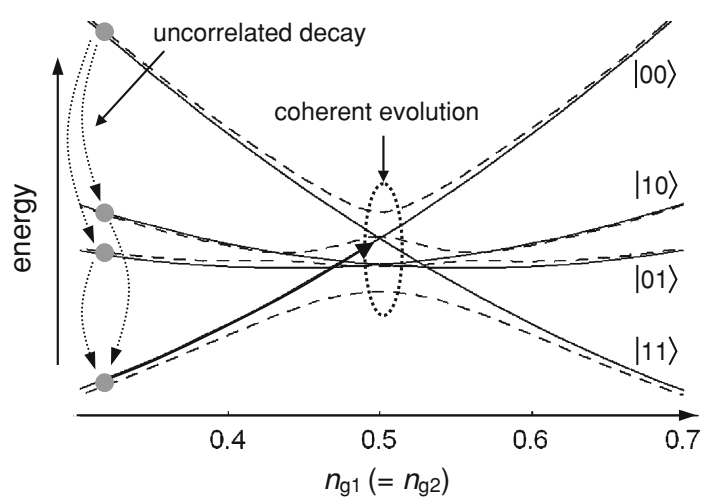

Fig. 9 Cross-sections of the energy bands through the point X in Fig. 8. Dashed lines: eigenenergies of Hamiltonian (12). Solid lines: electrostatic energies $E_{00}, E_{10}, E_{01}$, and $E_{11}$. Solid arrow along $E_{00}$ indicates non-adiabatic pulse shift of the system. Quantum state evolution involving all four basis states takes place at the gate voltages $n_{g 1}=n_{g 2}=0.5$ corresponding to the double degeneracy point $\mathrm{X}$ in Fig. 8: $E_{00}=E_{11}$ and $E_{10}=E_{01}$. Dotted lines on the left show qualitatively the decay of the excited states $(|11\rangle,|10\rangle$ and $|01\rangle)$ after the evolution (termination of the pulse). This decay gives rise to the pulse-induced probe currents in both probes

currents $I_{1,2}$ proportional to the probability $p_{1,2}(1)$ for each qubit to have a Cooper pair on it, regardless of the state of the other qubit, i.e., $I_{1} \propto p_{1}(1) \equiv\left|c_{2}\right|^{2}+\left|c_{4}\right|^{2}$ and $I_{2} \propto p_{2}(1) \equiv\left|c_{3}\right|^{2}+\left|c_{4}\right|^{2}$. Assuming the initial state at $t=0$ is $|00\rangle$, we derive for an ideal rectangular pulse shape of length $\Delta t$ the time evolution of these probabilities:

$$
p_{1,2}(1)=(1 / 4)\left[2-\left(1-\chi_{1,2}\right) \cos \{(\Omega+\epsilon) \Delta t\}-\left(1+\chi_{1,2}\right) \cos \{(\Omega-\epsilon) \Delta t\}\right]
$$

where $\Omega=\left(\left(E_{J 1}+E_{J 2}\right)^{2}+\left(E_{m} / 2\right)^{2}\right)^{1 / 2} / 2 \hbar, \epsilon=\left(\left(E_{J 1}-E_{J 2}\right)^{2}+\left(E_{m} / 2\right)^{2}\right)^{1 / 2} / 2 \hbar$ and $\chi_{1,2}=\left(E_{J 2,1}^{2}-E_{J 1,2}^{2}+E_{m}^{2} / 4\right) /\left(4 \hbar^{2} \Omega \epsilon\right)$.

Unlike the single qubit case, there are two frequencies present in the oscillation spectrum of the qubits: $\Omega+\epsilon$ and $\Omega-\epsilon$, both dependent on $E_{J 1}, E_{J 2}$ and $E_{m}$. Note that in the uncoupled situation $\left(E_{m}=0\right), \Omega \pm \epsilon=E_{J 1,2} / \hbar$ and each qubit oscillates with its own frequency $\omega_{1,2}$.

To measure coherent oscillations of coupled qubits, we drive the system to the point $X$ by an array of pulses and measure the pulse-induced currents $I_{1}$ and $I_{2}$. The pulse (solid arrow in Fig. 9) brings the system to the double degeneracy point, and the system evolves for the pulse duration time $\Delta t$, producing a superposed state Eq. 13 . After the pulse terminates, the system remains in the superposed state until it decays (dotted arrows in Fig. 9) to the ground state by emitting quasiparticles into the probe junctions.

The results for the coupled qubits are shown in Fig. 10. The observed spectral properties of the oscillations agree with the predictions of Eq. 14: there are two peaks in the spectrum and the peak positions are close to the expected frequencies $\Omega+\epsilon$ and $\Omega-\epsilon$ for the parameters $E_{J 1}=13.4 \mathrm{GHz}$ and $E_{J 2}=9.1 \mathrm{GHz}$ measured in the single qubit experiments, and $E_{m}=15.7 \mathrm{GHz}$ estimated from the independent dc 


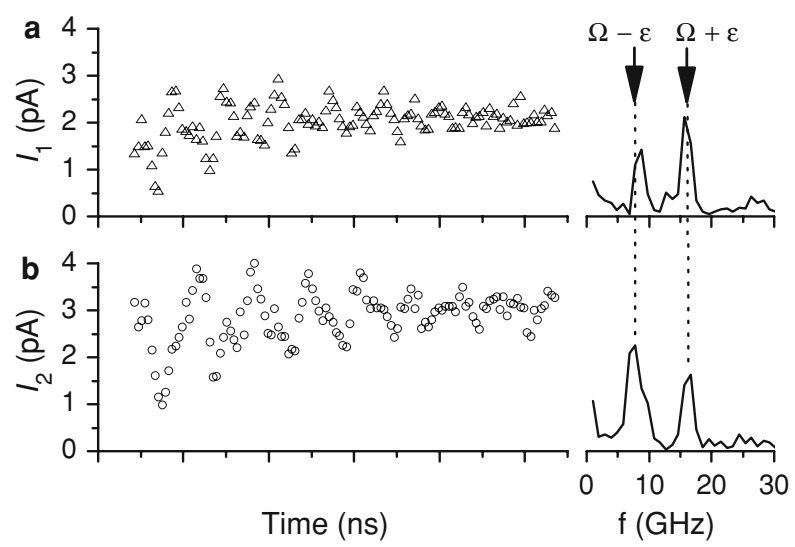

Fig. 10 Probe current oscillations in the first (a) and the second (b) qubit when the system is driven non-adiabatically to the double degeneracy point $X$ for the case $E_{J 1}=9.1 \mathrm{GHz}$ and $E_{J 2}=13.4 \mathrm{GHz}$. Right panels show the corresponding spectra obtained by Fourier transformation. Arrows and dashed lines indicate a theoretically expected position of the peaks

current-voltage characteristics measurement. Positions of the $\Omega+\epsilon$ and $\Omega-\epsilon$ peaks expected from Eq. 14 , are indicated by arrows and dashed lines. The decay time $\sim 0.6 \mathrm{~ns}$ of the coupled oscillations is shorter compared to the case of independent oscillations as should be expected, since qualitatively an extra decoherence channel is added to each qubit after coupling to its neighbor. A quantitative analysis of decoherence in coupled charge qubits is presented elsewhere [59].

Finally, we checked the dependence of the oscillation frequencies on $E_{J 1}$ controlled by a weak magnetic field (up to $20 \mathrm{Gs}$ ). As expected, we observed an anticrossing with a minimal gap at $E_{J 1}=E_{J 2}$ equal to $E_{m} / 2 h$. The observed dependence agrees with the prediction of Eq. 14 .

The observed quantum coherent dynamics of coupled qubits in the vicinity of the double-degeneracy point is clear evidence for the interaction of two qubits. The fact that the two qubits interact, in turn, implies also that they also become entangled during the course of coupled oscillations, although direct measurement of the degree of entanglement was not possible in this experiment. We have done numerical simulations of the entanglement evolution using different measures for the amount of entanglement: negativity, concurrence and entropy of formation [60]. Analytical calculation of the entropy of entanglement using a standard expression for the case of pure states [61] is presented in Ref. [58]. It has an oscillatory behavior and reaches unity at about $0.2 \mathrm{~ns}$. This means that the two qubits become maximally entangled at this instance. Our numerical simulations show that the amount of entanglement does not decrease significantly with a realistic pulse shape taken into account.

\subsection{Quantum logic gate}

The circuit described in Ref. [15], but with one more pulse gate, was also used for the demonstration of conditional gate operation [21]. Having two pulse gates enables 
Fig. 11 Ground state stability diagram of the coupled qubits. Point A is the operation point, and the white and black arrows represent pulses for input preparation and conditional gate operation, respectively

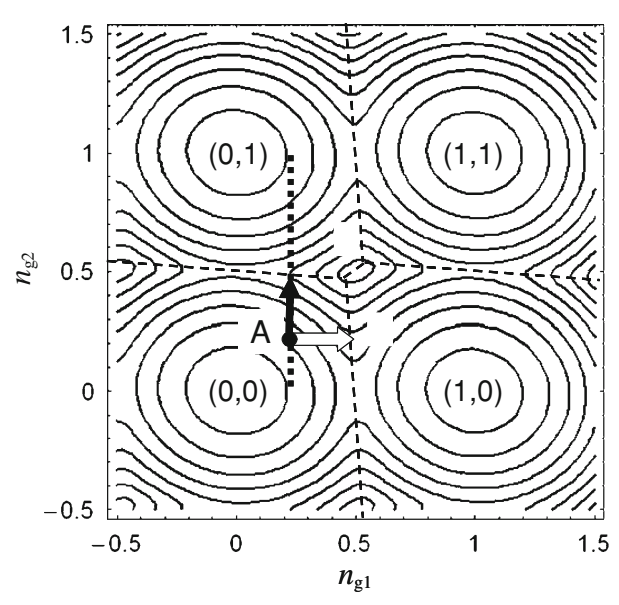

us to address each qubit individually. By utilizing the difference of the degeneracy condition between two pairs of the charge states, namely, $|00\rangle,|10\rangle$ and $|01\rangle,|11\rangle$, we can flip the state of the target qubit only when the control qubit is in the $|0\rangle$ state.

In the two-qubit charge basis $|00\rangle,|10\rangle,|01\rangle$ and $|11\rangle$, the Hamiltonian of the coupled charge qubit system is given by Eq. 12 with the same notations except that the pulse gate capacitances $C_{p 1}$ and $C_{p 2}$ contribute to the total capacitance of each Cooper pair box.

Figure 11 shows schematically the stability diagram of the coupled charge qubit system. Voltage pulses applied to pulse gates 1 and 2 move the system nonadiabatically along $n_{g 1}$ and $n_{g 2}$ axes, respectively. In our previous experiment to demonstrate quantum beating [15], we first set the operation point (point A) sufficiently far away from the double degeneracy point $\left(n_{g 1}, n_{g 2}\right)=(0.5,0.5)$ and brought the system nonadiabatically to the co-resonant point, where four eigenenergies become close with each other, and let the system evolve freely. For the conditional gate, however, we fix $n_{g} 1$ and operate along the dashed line in the figure.

Figure 12 shows the energy bands of the system along the dashed line in Fig. 11. Four energy bands can be regarded as two pairs of nearly independent single-qubit energy bands. For the lower two bands, the first qubit (control qubit) is always in the $|0\rangle$ state, while for the higher two bands, the first qubit is in the $|1\rangle$ state. Importantly, the charging energies of each of the two-level systems are degenerate at different $n_{g 2}$. This difference originates from the electrostatic coupling between the qubits and can be utilized for the conditional gate operation.

For the conditional gate operation, we apply a voltage pulse to pulse gate 2 so that it brings the system to the degeneracy point for the two lower bands (Fig. 12a). Suppose we start from the $|00\rangle$ state. Application of this pulse induces the oscillation between $|00\rangle$ and $|01\rangle$ states with maximum amplitude, as schematically shown by the Bloch sphere in Fig. 12b. By properly tuning the length of the pulse, we can stop the oscillation when the system is in the $|01\rangle$ state. By the same pulse, we obtain $|00\rangle$ state from the input state of $|01\rangle$. On the other hand, when the initial state is $|10\rangle$ or $|11\rangle$, the pulse induces oscillation between $|10\rangle$ and $|11\rangle$. However, because the system is 

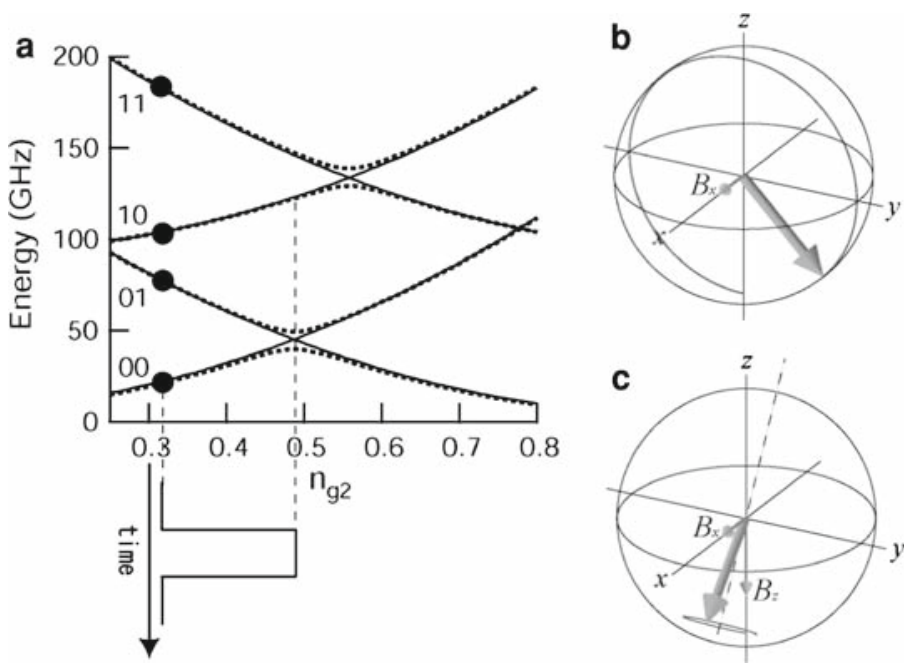

Fig. 12 a Energy bands calculated from Eq. 12. Dashed lines are the eigenenergies and solid lines are the charging energies of the states shown in the figure. They are plotted as functions of $n_{g 2}$, while $n_{g 1}$ is fixed as 0.18 . The rectangular-shape pulse represents the conditional gate operation. $\mathbf{b}, \mathbf{c}$ Single qubit Bloch sphere picture of the target qubit. When the control qubit is in state $|0\rangle$ (b), the operation pulse creates a resonance for the target qubit, hence Bloch vector rotates around the $x$-axis with maximum amplitude. When the control qubit is in state $|1\rangle(\mathbf{c})$, the operation pulse does not create a resonance, and there is a finite fictitious magnetic field along the $z$-axis, which leads to the suppressed oscillation amplitude

not brought to the degeneracy point for $|10\rangle$ and $|11\rangle$ states, the oscillation amplitude is suppressed due to the finite fictitious magnetic field along $z$ axis (Fig. 12c). The magnitude of this fictitious field is proportional to the coupling energy. Thus, for a given state of the target qubit, this pulse performs the conditional gate operation, i.e. the state of the target qubit is flipped only when the control qubit is in the $|0\rangle$ state.

In general, the input state $\alpha|00\rangle+\beta|10\rangle$ created by the first pulse applied to pulse gate 1 is transformed into state $\alpha|01\rangle+\beta|10\rangle$ by the second pulse applied to pulse gate 2 . There are two ways to control coefficients $\alpha$ and $\beta$ : either change $E_{J 1}$ by a magnetic field keeping $\Delta t_{1}$ constant [21], or change $\Delta t_{1}$ keeping $E_{J 1}$ constant [62]. The latter case is technically more difficult as the length of the first pulse is changed while the second pulse is fixed.

After we adjusted $\Delta t_{2}$ to have maximal $I_{2}$, we perform conditional gate operation for various input states and measure the output currents $I_{1} \propto|\beta|^{2}$ and $I_{1} \propto|\alpha|^{2}=1-|\beta|^{2}$ that are anti-correlated. Indeed, this anti-correlation between the two output currents seen in Fig. 13a, b is consistent with this expectation. Quantitatively, the modulation amplitude of the current of the target qubit agrees well with the result of the numerical simulation, which takes into account the finite rise and fall time of the pulse ( 40 ps) [21].

\section{Single artificial-atom lasing}

In this section we show how a charge qubit can be used as an artificial atom for the generation of coherent $\mathrm{CW}$ microwave radiation [63]. Indeed, charge qubits, like other 

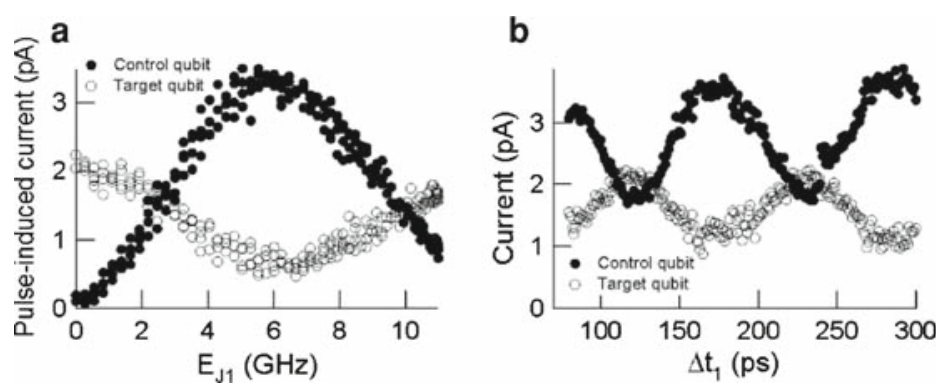

Fig. 13 Anti-correlation of the measured currents of the control and target qubits, as expected from the CNOT operation. a Pulse-induced currents of the control and target qubits as a function of $E_{J 1}$. b Pulseinduced currents of the control and target qubits as a function of $\Delta t_{1}$

types of qubits, behave like atoms: they have discrete energy levels and transitions may take place between them. These artificial atoms have certain advantages over the natural ones: (i) they can be fabricated with desired properties (energy gap, for example); (ii) their energy levels can be tuned in situ by external electrical or magnetic signals; (iii) they have stronger coupling to the electrical or magnetic fields due to their size. Such properties can be useful for various types of experiments, lasing being one of them.

For constructing a laser, three key components are required: an active quantum media, a resonator, and a mechanism of population inversion in the media. Our media is an artificial atom based on the charge qubit. Our resonator is a piece of a superconducting strip placed in between two grounded electrodes - a coplanar waveguide resonator. Finally, the population inversion in the artificial atom is created by the JQP current, a dc current that acts as pumping. The striking difference between this device and conventional lasers and masers is that its radiation is produced by one and the same atom coupled to the resonator.

Previous experiments $[64,65]$ with single natural atoms demonstrated the possibility of lasing for the strong coupling regime when the atom-cavity interaction time becomes shorter than the photon lifetime or the atom coherence time. Achieving this regime with single natural atoms seems rather difficult. On the other hand, quantum systems with artificial atoms allow one to easily make the interaction time much shorter than the coherence time, as it has been demonstrated previously [66,67]. Furthermore, controllable interaction with a single cavity mode together with a fast mechanism of population inversion allows one to realize a lasing regime with many photons generated by one and the same atom $[65,68,69]$.

The artificial-atom maser consists of a resonator and a charge qubit coupled to it (Fig. 14a). We fabricated a transmission-type half-wavelength coplanar-waveguide resonator using a 200-nm thick $\mathrm{Nb}$ film. It has a bare resonance frequency $\omega_{0} / 2 \pi=$ $9.889 \mathrm{GHz}$ and a quality factor $Q=7.6 \times 10^{3}$. The corresponding photon lifetime is $\tau_{p}=1.2 \times 10^{-7} \mathrm{~s}$ (the photon decay rate $\kappa=\tau_{p}^{-1}, \kappa / 2 \pi=1.3 \mathrm{MHz}$ ). The qubit is placed close to the end of the resonator where the electric field is nearly maximal.

Because quasiparticle states are also involved, it is convenient in this section to use notations that are slightly different from those used in the previous sections. We 
Fig. 14 a Schematics of the single artificial-atom maser. b Qubit energy band diagram and the lasing mechanism
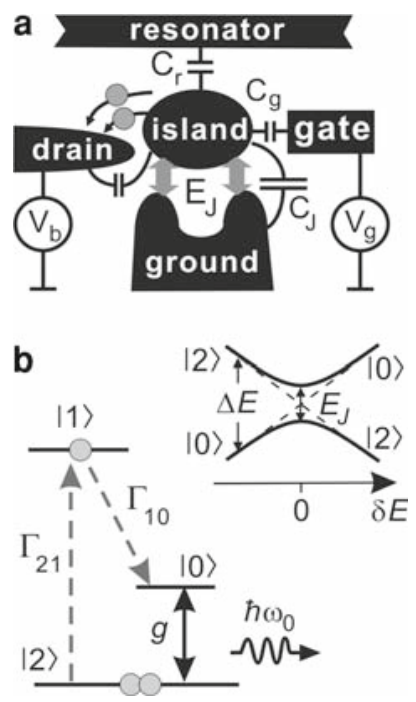

denote Cooper pair states of the qubit as $|0\rangle$ and $|2\rangle$ and the normalized gate charge $n_{g}=C_{g} V_{g} / e$. The qubit is characterized by the Josephson energy $E_{J}$ and the singleelectron charging energy $E_{c}$. The electrostatic energy difference $\delta E=4 E_{c}\left(n_{g}-1\right)$ between the two states is controlled by the gate voltage $V_{g}$ applied though the gate capacitance $C_{g}$. The qubit eigenenergy follows $\Delta E=\sqrt{\delta E^{2}+E_{J}^{2}}$ (top right panel of Fig. 14b). As the qubit is coupled to the resonator through an electric field $\left[\propto\left(a+a^{+}\right)\right]$, the Hamiltonian of the qubit-resonator system reads

$$
H=-\frac{1}{2}\left(\delta E \sigma_{z}+E_{J} \sigma_{x}\right)+\hbar \omega_{0}\left(a^{+} a+\frac{1}{2}\right)+\hbar g_{0}\left(a+a^{+}\right) \sigma_{z} .
$$

The first, second and third terms describe the qubit; resonator and qubit-resonator coupling; $a^{+}$and $a$ are the photon creation and annihilation operators, respectively, and $g_{0}$ is the coupling strength. The value of $g_{0} / 2 \pi$ is found to be $80 \mathrm{MHz}$ from the fitting of the dispersion curve of transmission through the resonator when the qubit is biased at $\delta E=0 \delta$. This is consistent with the expectation $g_{0} / 2 \pi=\left(e V_{0} / 2 \pi \hbar\right)\left(C_{r} / C_{\Sigma}\right)$, where $V_{0}=\sqrt{\hbar \omega_{0} / C_{0}} \sim 1.8 \mu \mathrm{V}$ is the zero-point fluctuation voltage of the resonator with the total capacitance $C_{0}=2 \mathrm{pF}$. The observed anti-crossing demonstrates that the qubit is indeed coherently coupled to the resonator [70].

To create population inversion in the qubit we introduce a drain electrode connected to the island via a tunnel junction with the resistance $R_{b}$ of $1.0 \mathrm{M} \Omega$ (Fig. 14a, b). The drain electrode is voltage biased at a voltage $V_{b}$ above $\left(2 \Delta+E_{c}\right) / e$, which is required to extract two electrons from the island by breaking a Cooper pair $(\Delta / h \approx 55 \mathrm{GHz})$. As a result, $|2\rangle$ decays into $|0\rangle$ via two sequential single-electron tunnelling events in the incoherent process $|2\rangle \rightarrow|1\rangle \rightarrow|0\rangle$ (bottom left panel of Fig. 14b), and the 'atom' is pumped into $|0\rangle$ state. At $\delta E=0$, a Cooper pair tunnels resonantly across the Josephson junction from the reservoir to the island $(|0\rangle \rightarrow|2\rangle)$. Thus, the Josephson- 
quasiparticle cycle involving the three charge states continues and results in a pronounced current peak. For $\delta E \gg E_{J}$ the upper eigenstate of the qubit is nearly $|0\rangle$ state, and the single-electron tunnelling process creates population inversion with an effective rate $\gamma \approx V_{b} / 2 e R_{b} \approx 2 \times 10^{9} \mathrm{~s}^{-1}(\gamma / 2 \pi \approx 320 \mathrm{MHz})$.

When $\Delta E$ is adjusted to $\hbar \omega_{0}$ the photon number in the qubit-resonator system is not conserved. The basis states of the system described by the Hamiltonian of Eq. 15 are represented as $|n, N\rangle$, where $n$ is the number of electrons and $N$ is the number of photons in the resonator. The energy quantum of the qubit is transferred into the resonator as a photon, accompanied by a Cooper pair tunnelling across the Josephson junction $(|0, N\rangle \rightarrow|2, N+1\rangle$, starting from $N=0)$. The cycle proceeds repeatedly, and $\mathrm{N}$ in the resonator increases until it reaches the balance between the photon generation and loss. The qubit-resonator coupling grows with $N$ as $g_{0} \sqrt{N+1}$. The photon field stimulates the photon generation process, which is analogous to the stimulated emission in conventional lasers. In contrast to conventional lasers with many atoms, emission observed from the single atom coupled to the single-mode cavity immediately implies "thresholdless" lasing [65,68]. In our lasing mechanism, the photon flux is related to the current flowing through the qubit. Therefore, an additional feature related to lasing is expected in the dc current [71].

In Fig. 15, emission power spectral density from the resonator (upper panel) is shown together with the current through the qubit (lower panel) as a function of $\delta E$ at $V_{b}=0.65 \mathrm{mV}$. The observed current peak is due to the JQP process. On the right slope of the JQP peak ( $\delta E>0$; the emission side), two small current peaks appear. At the same time, we observe strong emission shown as two "hot spots" in the upper panel. The position of the first current peak and the hot spot corresponds to $\delta E / h \sim 7 \pm 2 \mathrm{GHz}$. Although the hot spot is rather broad, its position is consistent with the condition $\Delta E=\hbar \omega_{0}$. One possible interpretation of the presence of the second hot spot is the two-photon resonance [71]. Note that the emission takes place only

Fig. 15 Emission power spectrum from the resonator (upper panel) together with the current $I$ through the qubit (lower panel) as a function of $\delta E$ and $n_{g}$. Emission is seen as two "hot spots", and the corresponding current peaks appear on the right slope of the JQP peak $(\delta E>0)$

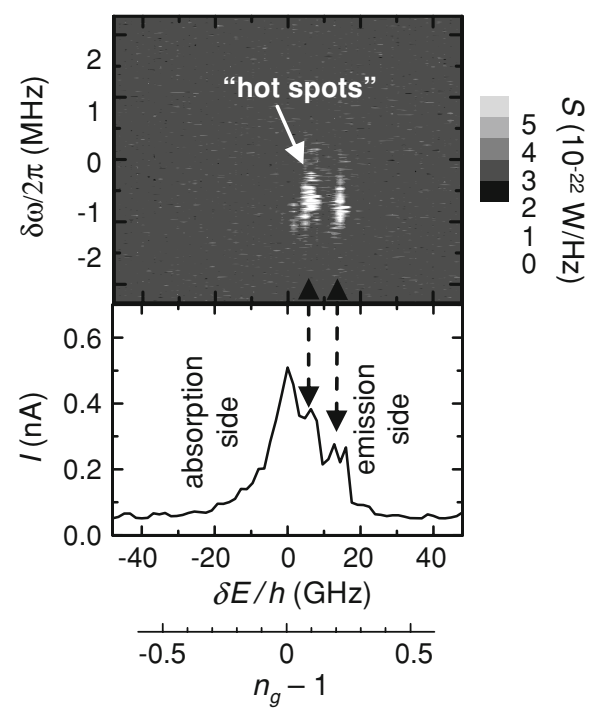


Fig. 16 Output power spectrum $S$ (grayscale map in log scale) as a function of the driving power for the detuning frequency $\delta \omega_{\text {drive }} / 2 p i=-0.5 \mathrm{MHz}$. The spectrum gets as narrow as the measurement bandwidth $(100 \mathrm{kHz})$ when $N^{*}>\sim 1$

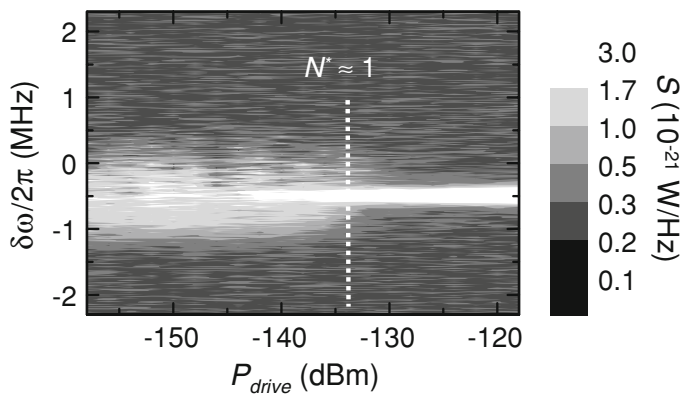

when the drain electrode is biased in the range $0.57 \mathrm{mV} \leq V_{b} \leq 0.71 \mathrm{mV}$ where the JQP current peak is observed.

At the hot spot, the emission peak is unstable, showing low-frequency fluctuations, which can be naturally attributed to the low frequency charge noise. Nonetheless, the average emission spectrum width $0.7 \mathrm{MHz}$ is narrower than $\kappa$ and $\gamma$, which strongly supports lasing mechanism. The total emission power within the hot spot is estimated to be $W=7 \times 10^{-16} \mathrm{~W}$ corresponding to $N=2\left(W / \hbar \omega_{0}\right) \tau_{p} \approx 30$ photons in the resonator (the factor 2 comes from the equal probability for the photons to escape through each end of the resonator). This number may be an underestimate as the resonator internal loss is not accounted for. The JQP current at its peak ( $I \sim 0.4-0.2 \mathrm{nA})$ generates less than $I / 2 e \sim 10^{9} \mathrm{~s}^{-1}$ excited states, which is comparable to the photon generation rate $N / \tau_{p}>2.5 \times 10^{8} \mathrm{~s}^{-1}$. The high photon generation efficiency satisfies the main criterion for "thresholdless" lasing.

Next we study the emission spectrum under the external microwave drive, expecting "injection locking" effects [72]. Figure 16 shows the emission power spectrum as a function at the driving power $P_{\text {drive }}$ and detuning $\delta \omega$. The emission peak is broad at low driving power $\left(P_{\text {drive }}<-134 \mathrm{dBm}\right)$. However, when $P_{\text {drive }}$ exceeds the level of one photon in the resonator, the emission line shrinks to the drive frequency with the width limited by the measurement bandwidth $(100 \mathrm{kHz})$. The effect of frequency stabilization and emission narrowing known in laser physics as "injection locking" additionally proves lasing action.

\section{Summary}

In summary, we have performed a number of pioneering experiments demonstrating

a) quantum coherence in a solid-state qubit;

b) coherent dynamics of two coupled charge qubits;

c) quantum logic gate;

d) single-shot readout of a charge qubit

e) lasing effect in a single qubit coupled to a resonator.

We have also studied mechanisms of decoherence in charge qubits. Despite a decade of intensive research, the quantum computer still remains a dream. Many hurdles are to be overcome, decoherence being the major one. Nonetheless, even now the 
superconducting qubits have many potential applications including lasing, creation of metamaterials, on-chip quantum optics, quantum-limited detectors to mention a few.

Open Access This article is distributed under the terms of the Creative Commons Attribution Noncommercial License which permits any noncommercial use, distribution, and reproduction in any medium, provided the original author(s) and source are credited.

\section{References}

1. Nielsen, M.A., Chuang, I.L.: Quantum Computation and Quantum Information. Cambridge Univ. Press, Cambridge (2000)

2. Clark, R.G. (ed.): Experimental Implementation of Quantum Computation. Rinton Press, Princeton (2001)

3. Averin, D.V.: Quantum computing and quantum measurement with mesoscopic Josephson junctions. Fortschr. Phys. 48, 1055-1074 (2000)

4. Makhlin, Y., Schön, G., Shnirman, A.: Quantum-state engineering with Josephson-junction devices. Rev. Mod. Phys. 73, 357-400 (2001)

5. Nakamura, Y., Pashkin, Yu.A., Tsai, J.S.: Coherent control of macroscopic quantum states in a singlecooper-pair box. Nature 398, 786-788 (1999)

6. Vion, D., Aasime, A., Cottet, A., Joyez, P., Pothier, H., Urbina, C., Esteve, D., Devoret, M.H.: Manipulating the quantum state of an electrical circuit. Science 296, 886-889 (2002)

7. Duty, T., Gunnarsson, D., Bladh, K., Delsing, P.: Coherent dynamics of a Josephson charge qubit. Phys. Rev. B 69, 140503 (2004)

8. Chiorescu, I., Nakamura, Y., Harmans, C.J.P.M., Mooij, J.E.: Coherent quantum dynamics of a superconducting flux qubit. Science 299, 1869-1871 (2003)

9. Yu, Y., Han, S., Chu, X., Chu, S.-I., Wang, Z.: Coherent temporal oscillations of macroscopic quantum states in a Josephson junction. Science 296, 889-892 (2002)

10. Martinis, J.M., Nam, S., Aumentado, J., Urbina, C.: Rabi oscillations in a large Josephson-junction qubit. Phy. Rev. Lett. 89, 117901 (2002)

11. Hayashi, T., Fujisawa, T., Cheong, H.D., Jeong, Y.H., Hirayama, Y.: Coherent manipulation of electronic states in a double quantum dot. Phys. Rev. Lett. 91, 226804 (2003)

12. Petta, J.R., Johnson, A.C., Taylor, J.M., Laird, E.A., Yacoby, A., Lukin, M.D., Marcus, C.M., Hanson, M.P., Gossard, A.C.: Coherent manipulation of coupled electron spins in semiconductor quantum dots. Science 309, 2180-2184 (2005)

13. Koppens, F.H.L., Buizert, C., Tielrooij, K.J., Vink, I.T., Nowack, K.C., Meunier, T., Kouwenhoven, L.P., Vandersypen, L.M.K.: Driven coherent oscillations of a single electron spin in a quantum dot. Nature 442, 766-771 (2006)

14. Pioro-Ladriere, M., Obata, T., Tokura, Y., Shin, Y.-S., Kubo, T., Yoshida, K., Taniyama, T., Tarucha, S.: Electrically driven single-electron spin resonance in a slanting Zeeman field. Nat. Phys. 4, 776779 (2008)

15. Pashkin, Yu.A., Yamamoto, T., Astafiev, O., Nakamura, Y., Tsai, J.S.: Quantum oscillations in two coupled charge qubits. Nature 421, 823-826 (2003)

16. Berkley, A.J., Xu, H., Ramos, R.C., Gubrud, M.A., Strauch, F.W., Johnson, P.R., Anderson, J.R., Dragt, A.J., Lobb, C.J., Wellstood, F.C.: Entangled macroscopic quantum states in two superconducting qubits. Science 300, 1548-1550 (2003)

17. Izmalkov, A., Grajcar, M., Il'ichev E., Wagner, Th., Meyer, H.-G., Smirnov, A.Yu., Amin, M.H.S., van den Brink, A.M., Zagoskin, A.M.: Evidence for entangled states of two coupled flux qubits. Phys. Rev. Lett. 93, 037003 (2004)

18. McDermott, R., Simmonds, R.W., Steffen, M., Cooper, K.B., Cicak, K., Osborn, K.D., Oh, S., Pappas, D.P., Martinis, J.M.: Simultaneous state measurement of coupled Josephson phase qubits. Science 307, 1299-1302 (2005)

19. Majer, J.B., Paauw, F.G., Ter Haar, A.C.J., Harmans, C.J.P.M., Mooij, J.E.: Spectroscopy on two coupled superconducting flux qubits. Phys. Rev. Lett. 94, 090501 (2005)

20. Hime, T., Reichardt, P.A., Plourde, B.L.T., Robertson, L.T., Wu, C.-E., Ustinov, A.V., Clarke, J.: Solidstate qubits with current-controlled coupling. Science 314, 1427-1429 (2006) 
21. Yamamoto, T., Pashkin, Yu.A., Astafiev, O., Nakamura, Y., Tsai, J.S.: Demonstration of conditional gate operation using superconducting charge qubits. Nature 425, 941-944 (2003)

22. Steffen, M., Ansmann, M., Bialczak, R.C., Katz, N., Neeley, M., Weig, E.M., Cleland, A.N., Martinis, J.M.: Measurement of the entanglement of two superconducting qubits via state tomography. Science 313, 1423-1425 (2006)

23. Plantenberg, J.H., de Groot, P.C., Harmans, C.J.P.M., Mooij, J.E.: Demonstration of controlled-NOT quantum gates on a pair of superconducting quantum bits. Nature 447, 836-839 (2007)

24. DiVincenzo, D.P.: The physical implementation of quantum computation. Fortschr. Phys. 48, 771-783 (2000)

25. Dolan, G.J.: Offset masks for lift-off photoprocessing. Appl. Phys. Lett. 31, 337-339 (1977)

26. Büttiker, M.: Zero-current persistent potential drop across small-capacitance Josephson junctions. Phys. Rev. B 36, 3548-3555 (1987)

27. Bouchiat, V., Vion, D., Joyez, P., Esteve, D., Devoret, M.H.: Quantum coherence with a single Cooper pair. Phys. Scripta T 76, 165-170 (1998)

28. Likharev, K.K., Zorin, A.B.: Theory of the Bloch-wave oscillations in small Josephson junctions. J. Low Temp. Phys. 59, 347-382 (1985)

29. Nakamura, Y., Chen, C.D., Tsai, J.S.: Spectroscopy of energy-level splitting between two macroscopic quantum states of charge coherently superposed by Josephson coupling. Phys. Rev. Lett. 79, 23282331 (1997)

30. Fulton, T.A., Gammel, P.L., Bishop, D.J., Dunkleberger, L.N., Dolan, G.J.: Observation of combined Josephson and charging effects in small tunnel junction circuits. Phys. Rev. Lett. 63, 1307-1310 (1989)

31. Averin, D.V., Aleshkin, V.Ya.: Resonance tunneling of Cooper pairs in a system of small Josephson junctions. JETP Lett. 50, 367-369 (1989)

32. Nakamura, Y., Tsai, J.S.: Quantum coherence in a superconducting single-electron box. In: Friedman, J., Han, S. (eds.) Exploring the Quantum/Classical Frontier: Recent Advances in Macroscopic Quantum Phenomena, pp. 97-129. Nova Science, New York (2003)

33. Nakamura, Y., Tsai, J.S.: Quantum-state control with a single-cooper-pair box. J. Low Temp. Phys. 118, 765-779 (2000)

34. Barone, A., Paterno, G.: Physics and Applications of the Josephson Effect. Wiley, New York (1982)

35. Wallraff, A., Schuster, D.I., Blais, A., Frunzio, L., Majer, J., Girvin, S.M., Schoelkopf, R.J.: Approaching unit visibility for control of a superconducting qubit with dispersive readout. Phys. Rev. Lett. 95, 060501 (2005)

36. Siddiqi, I., Vijay, R., Metcalfe, M., Boaknin, E., Frunzio, L., Schoelkopf, R.J., Devoret, M.H.: Dispersive measurements of superconducting qubit coherence with a fast, latching readout. Phys. Rev. B 73, 054510 (2006)

37. Sillanpää, M.A., Lehtinen, T., Paila, A., Makhlin, Yu., Roschier, L., Hakonen, P.J.: Direct observation of macroscopic quantum renormalization of capacitance. Phys. Rev. Lett. 95, 206806 (2005)

38. Duty, T., Johansson, G., Bladh, K., Gunnarsson, D., Wilson, C., Delsing, P.: Observation of quantum capacitance in the Cooper-pair transistor. Phys. Rev. Lett. 95, 206807 (2005)

39. Nakamura, Y., Pashkin, Yu.A., Tsai, J.S.: Rabi oscillations in a Josephson-junction charge two-level system. Phys. Rev. Lett. 87, 246601 (2001)

40. Astafiev, O., Pashkin, Yu.A., Yamamoto, T., Nakamura, Y., Tsai, J.S.: Single-shot measurement of the Josephson charge qubit. Phys. Rev. B 69, 180507 (2004)

41. Nakamura, Y., Pashkin, Yu.A., Yamamoto, T., Tsai, J.S.: Charge echo in a Cooper-pair box. Phys. Rev. Lett. 88, 047901 (2002)

42. Slichter, C.P.: Principles of Magnetic Resonance, 3rd edn. Springer, New York (1990)

43. Zimmerly, G., Eiles, T.M., Kautz, R.L., Martinis, J.M.: Noise in the Coulomb blockade electrometer. Appl. Phys. Lett. 61, 237-239 (1992)

44. Verbrugh, S.M., Benhamadi, M.L., Visscher, E.H., Mooij, J.E.: Optimization of island size in single electron tunneling devices: Experiment and theory. J. Appl. Phys. 78, 2830-2836 (1995)

45. Zorin, A.B., Ahlers, F.J., Niemeyer, J., Weimann, T., Wolf, H., Krupenin, V.A., Lotkhov, S.V.: Background charge noise in metallic single-electron tunneling devices. Phys. Rev. B 53, 1368213687 (1996)

46. Lehnert, K.W., Bladh, K., Spietz, L.F., Gunnarsson, D., Schuster, D.I., Delsing, P., Schoelkopf, R.J.: Measurement of the excited-state lifetime of a microelectronic circuit. Phys. Rev. Lett. 90, 027002 (2003) 
47. Ithier, G., Collin, E., Joyez, P., Meeson, P.J., Vion, D., Esteve, D., Chiarello, F., Shnirman, A., Makhlin, Y., Schriefl, J., Schön, G.: Decoherence in a quantum bit superconducting circuit. Phys. Rev. B 72, 134519 (2005)

48. Metcalfe, M.B., Boaknin, E., Manucharyan, V., Vijay, R., Siddiqi, I., Rigetti, C., Frunzio, L., Schoelkopf, R.J., Devoret, M.H.: Measuring the decoherence of a quantronium qubit with the cavity bifurcation amplifier. Phys. Rev. B 76, 174516 (2007)

49. Schreier, J.A., Houck, A.A., Koch, J., Schuster, D.I., Johnson, B.R., Chow, J.M., Gambetta, J.M., Majer, J., Frunzio, L., Devoret, M.H., Girvin, S.M., Schoelkopf, R.J.: Suppressing charge noise decoherence in superconducting charge qubits. Phys. Rev. B 77, 180502 (2008)

50. Schoelkopf, R.J., Clerk, A.A., Girvin, S.M., Lehnert, K.W., Devoret, M.: Qubits as spectrometers of quantum noise. In: Nazarov, Y.V. (ed.) Quantum Noise in Mesoscopic Physics, pp. 175-203. Kluwer, Amsterdam (2003)

51. Shnirman, A., Schön, G., Martin, I., Makhlin, Yu.: Low- and high-frequency noise from coherent two-level systems. Phys. Rev. Lett. 94, 127002 (2005)

52. Astafiev, O., Pashkin, Yu.A., Yamamoto, T., Nakamura, Y., Tsai, J.S.: Temperature square dependence of the low frequency $1 / \mathrm{f}$ charge noise in the Josephson junction qubits. Phys. Rev. Lett. 96, 137001 (2006)

53. Averin, D.V., Bruder, C.: Variable electrostatic transformer: Controllable coupling of two charge qubits. Phys. Rev. Lett. 91, 057003 (2003)

54. You, J.Q., Tsai, J.S., Nori, F.: Controllable manipulation and entanglement of macroscopic quantum states in coupled charge qubits. Phys. Rev. B 68, 024510 (2003)

55. Lantz, J., Wallquist, M., Shumeiko, V.S., Wendin, G.: Josephson junction qubit network with currentcontrolled interaction. Phys. Rev. B 70, 140507 (2004)

56. Yamamoto, T., Watanabe, M., You, Q.J., Pashkin, Yu.A., Astafiev, O., Nakamura, Y., Nori, F., Tsai, J.S.: Spectroscopy of superconducting charge qubits coupled by a Josephson inductance. Phys. Rev. B 77, 064505 (2008)

57. Pothier, H., Lafarge, P., Urbina, C., Esteve, D., Devoret, M.H.: Single-electron pump based on charging effects. Europhys. Lett. 17, 249-254 (1992)

58. Pashkin, Yu.A., Yamamoto, T., Astafiev, O., Nakamura, Y., Averin, D.V., Tilma, T., Nori, F., Tsai, J.S.: Coherent manipulation of coupled Josephson charge qubits. Physica C 426-431, 15521560 (2005)

59. Rabenstein, K., Averin, D.V.: Decoherence in two coupled qubits. Turk. J. Phys. 27, 313-322 (2003) [cond-mat/0310193]

60. Pashkin, Yu.A., Tilma, T., Averin, D.V., Astafiev, O., Yamamoto, T., Nakamura, Y., Nori, F., Tsai, J.S.: Entanglement of two coupled charge qubits. Int. J. Quantum Inform. 1, 421-426 (2003)

61. Bennett, C.H., Bernstein, H.J., Popescu, S., Schumacher, B.: Concentrating partial entanglement by local operations. Phys. Rev. A 53, 2046-2052 (1996)

62. Yamamoto, T., Pashkin, Yu.A., Astafiev, O., Nakamura, Y., Tsai, J.S.: Conditional gate operation in superconducting charge qubits. In: Ruggiero, B., et al. (eds.) Quntum Computation in Solid State Systems, pp. 10-18. Springer, New York (2006)

63. Astafiev, O., Inomata, K., Niskanen, A.O., Yamamoto, T., Pashkin, Yu.A., Nakamura, Y., Tsai, J.S.: Single artificial-atom lasing. Nature 449, 588-590 (2007)

64. Walther, H., Varcoe, B.T.H., Englert, B.-G., Becker, T.: Cavity quantum electrodynamics. Prog. Phys. 69, 1325-1382 (2006)

65. McKeever, J., Boca, A., Boozer, A.D., Buck, J.R., Kimble, H.J.: Experimental realization of a one-atom laser in the regime of strong coupling. Nature 425, 268-271 (2003)

66. Reithmaier, J.P., Sek, G., Löffler, A., Hofmann, C., Kuhn, S., Reitzenstein, S., Keldysh, L.V., Kulakovskii, V.D., Reinecke, T.L., Forchel, A.: Strong coupling in a single quantum dot-semiconductor microcavity system. Nature 432, 197-200 (2004)

67. Yoshie, T., Scherer, A., Hendrickson, J., Khitrova, G., Gibbs, H.M., Rupper, G., Ell, C., Shchekin, O.B., Deppe, D.G.: Vacuum Rabi splitting with a single quantum dot in a photonic crystal nanocavity. Nature 432, 200-203 (2004)

68. Rice, P.R., Carmichael, H.J.: Photon statistics of a cavity-QED laser: a comment on the laser-phasetransition analogy. Phys. Rev. A 50, 4318-4329 (1994)

69. Mu, Y., Savage, C.M.: One-atom lasers. Phys. Rev. A 46, 5944-5954 (1992) 
70. Wallraff, A., Schuster, D.I., Blais, A., Frunzio, L., Huang, R.-S., Majer, J., Kumar, S., Girvin, S.M., Schoelkopf, R.J.: Strong coupling of a single photon to a superconducting qubit using circuit quantum electrodynamics. Nature 431, 162-167 (2004)

71. Rodrigues, D.A., Imbers, J., Armour, A.D.: Quantum dynamics of a resonator driven by a superconducting single-electron transistor: a solid-state analogue of the micromaser. Phys. Rev. Lett. 98, 067204 (2008)

72. Siegman, A.E.: Lasers. University Science Books, Mill Valley (1986) 